\title{
Multiplicity Study of Exoplanet Host Stars: the HD 3651 AB System
}

\author{
M. Mugrauer ${ }^{1} \dagger$, A. Seifahrt ${ }^{2}$, R. Neuhäuser ${ }^{1}$, \\ T. Mazeh ${ }^{3}$, and T. Schmidt ${ }^{1}$ \\ ${ }^{1}$ Astrophysikalisches Institut, Universität Jena, Schillergäßchen 2-3, 07745 Jena, Germany \\ ${ }^{2}$ European Southern Observatory, Karl-Schwarzschild-Str. 2, 85748 Garching, Germany \\ ${ }^{3}$ Tel Aviv University, Tel Aviv 69978, Israel
}

\begin{abstract}
We report on new results of our multiplicity study and present our follow-up observations of the recently detected faint companion $\mathrm{HD} 3651 \mathrm{~B}$, the first directly-imaged $\mathrm{T}$ dwarf companion of an exoplanet host star.
\end{abstract}

Keywords. (stars:) binaries: visual, stars: low-mass, brown dwarfs

\section{Introduction}

In the course of our observations to search for wide faint companions to planet host stars, carried out in the infrared $(H$-band) with SofI at the NTT and UFTI at the UKIRT, we could already find several new companions (Mugrauer et al. 2004a, 2004b, 2005, 2006b). Most of these objects are low-mass stars with masses which range from the substellar-stellar mass border at about $0.075 \mathrm{M}_{\odot}$ up to about $0.5 \mathrm{M}_{\odot}$.

Two companions turned out to be white dwarfs, whose true nature could be revealed with spectrophotometric measurements. Gl 86 B is the first known close white dwarf companion of an exoplanet host star, separated from its primary by only 20 AU (Mugrauer \& Neuhäuser 2005, Mugrauer et al. 2006a). Its white dwarf nature could also be confirmed by combining radial velocity data with NACO astrometry, obtained during the last few years (Lagrange et al. 2006). These observations will finally constrain the orbital parameters of the companion and also allow to determine its mass. A first estimate shows that the white dwarf companion $\left(\mathrm{m} \sim 0.5 \mathrm{M}_{\odot}\right)$ revolves around the planet host star $\mathrm{Gl} 86 \mathrm{~A}$ on an eccentric $e=0.4$ orbit with a semi-major axis of only 18.4 AU. If we assume that the system was about 1.4 times closer before the mass loss of the white dwarf progenitor star (Weidemann 2000), such a close companion yields only a small long-time stable region for planets (only 1.7 AU, Holman \& Wiegert 1997). The same holds for the size of the protoplanetary disk in which the planet formation process took place (Pichardo et al. 2005), posing a problem to the theory of planet formation. Nevertheless, a massive planet $\left(\mathrm{m} \sin (i)=4 \mathrm{M}_{\mathrm{Jup}}\right.$, Queloz et al. 2000) had been formed in this environment and finally also survived the post-main-sequence evolution of the white dwarf progenitor star.

A further white dwarf companion could be detected around the planet host star HD 27442 A. The white dwarf nature of HD 27442 B (WDS binary, Worley \& Douglas 1997 ) could be revealed with SofI photometry and ISAAC and FORS spectroscopy (Mugrauer et al. 2006, submitted to $A \mathscr{E} A$ ). This companion is separated from the planet host star by already $\sim 240 \mathrm{AU}$, hence this is a less challenging environment for planet formation than the $\mathrm{Gl} 86 \mathrm{AB}$ system. If planets can form in a $\mathrm{Gl} 86 \mathrm{AB}$ like system and also survive the post-main-sequence evolution of the white dwarf progenitor star, planets should also

$\dagger$ markus@astro.uni-jena.de 
exist in wider such evolved stellar systems with white dwarf companions. However, the HD $27442 \mathrm{AB}$ system is a special case because of the nature of the planet host star which is an already evolved subgiant (Butler et al. 2001). With its white dwarf companion and the subgiant planet host star, the HD $27442 \mathrm{AB}$ system is the most evolved planet-hosting stellar system presently known.

\section{The HD 3651 AB system}

Recently, we reported the detection of a new very faint companion $43^{\prime \prime}$ northwest of the planet host star HD 3651 A (Mugrauer et al. 2006c). This companion, HD 3651 B, was followed by us in four observing epochs with UFTI at UKIRT and SofI at NTT, clearly confirming that it shares the proper motion of the nearby planet host star. The averaged SofI and UFTI $H$-band photometry of the companion is $H=16.75 \pm 0.16 \mathrm{mag}$, i.e., this is the faintest co-moving companion of an exoplanet host star presently known. HD $3651 \mathrm{~B}$ is not detected in the visible (POSS plates) but appears only as a faint source in the infrared, i.e., it is a cool brown dwarf companion, the first of its kind directly imaged as a companion of an exoplanet host star. The $H$-band photometry is consistent with a $\mathrm{T}$ dwarf of spectral type $\mathrm{T} 7$ to T8 $(800-900 \mathrm{~K})$. HD $3651 \mathrm{~B}$ is one of the faintest $\mathrm{T}$ dwarfs ever imaged.
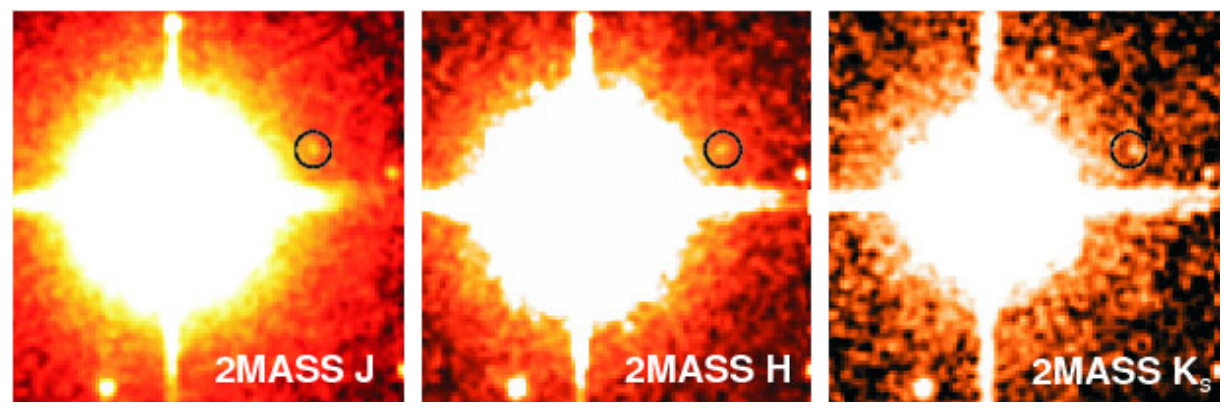

Figure 1. The 2MASS $J_{-}, H$-, and $K_{\mathrm{S}}$-band Atlas images. The expected position of HD $3651 \mathrm{~B}$ is at the center of the black circles. We detected a faint source in 2MASS $J_{-}$, and $H$-band images, which is not visible in the $2 \mathrm{MASS} K_{\mathrm{S}}$-band image. This might be the co-moving companion HD $3651 \mathrm{~B}$, but could be spurious.

During the astrometrical analysis process of our imaging data we also studied the 2MASS $J_{-}, H$-, and $K_{\mathrm{S}}$-band images (Figure 1 ) in order to check whether the companion is detected in the 2MASS data, providing additional astrometric and photometric data. Indeed, there is a very faint noisy source $(S / N<5)$ which can be found in the 2MASS $J$ - and $H$-band images exactly at the expected position of HD $3651 \mathrm{~B}$. In the $K_{\mathrm{S}}$-band there is a source detected slightly westward of the expected position. This faint source is not listed in the 2MASS Point Source Catalog (Skrutskie et al. 2006). It might be the companion, but could also be spurious. Due to the faintness of this source, and because there are several comparable bright speckles detected in the 2MASS images, we did not use the 2MASS data for astrometric or photometric analysis. In contrast, in our four images obtained with UFTI and SofI HD 3651 B is clearly detected, hence accurate astrometric and photometric measurements are possible.

In addition to our UFTI and SofI imaging we obtained follow-up $J-, H$-, and $K$-band imaging data of the new companion with OMEGA-CASS at the 3.5-m telescope of the Calar Alto Observatory (southern Spain). The companion was observed $9.8 \mathrm{~min}$ in $J$ - and $H$-, and $35.5 \mathrm{~min}$ in $K$-band. The $J$ - and $H$-band OMEGA-CASS images are both the 

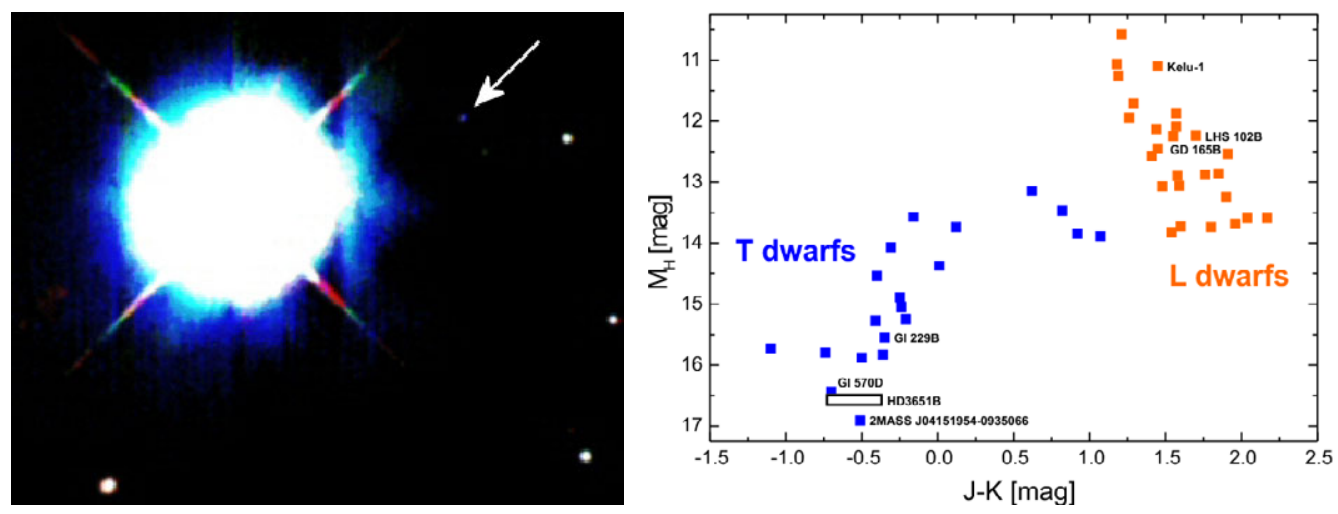

Figure 2. Left: This is a $J$ - (blue), $H$ - (green), and $K$-band (red) color composite-image of the exoplanet host star HD 3651 A (bright star in the center) taken with the infrared camera OMEGA-CASS at the 3.5-m telescope at Calar Alto Observatory. The infrared imaging is carried out in the high resolution mode $(0.2 \mathrm{arcsec} / \mathrm{pixel})$. The detected faint co-moving companion HD $3651 \mathrm{~B}$ is marked with an arrow. Right: HD $3651 \mathrm{~B}$ in the $(J-K)-\mathrm{M}_{H}$ diagram together with comparison brown dwarfs from Knapp et al. (2004). HD $3651 \mathrm{~B}$ is one of the faintest $\mathrm{T}$ dwarfs presently known.

average of 10 frames, each the average of $491^{\text {s }} .2$ integrations. The $K$-band image is the average of 29 frames, each the average of 49 1.5 integrations. We measure the infrared photometry of HD $3651 \mathrm{~B}$ in all OMEGA-CASS images and obtain: $J=15.98 \pm 0.12$ mag, $H=16.80 \pm 0.08 \mathrm{mag}$, and $K=16.53 \pm 0.13 \mathrm{mag}$. The companion can be plotted then in a color-magnitude diagram (Figure 2) together with comparison brown dwarfs from Knapp et al (2004). The OMEGA-CASS photometry confirms our conclusion that the companion is a late $\mathrm{T}$ dwarf. Its photometry is fully consistent with late $\mathrm{T}$ dwarfs like Gl570 D (Burgasser et al. 2000) or 2MASS J0415 (Burgasser et al. 2002). Our spectral type estimate was most recently confirmed by Luhman et al. (2006) and Burgasser (2006).

\section{References}

Burgasser, A.J., et al., 2000, ApJL, 531, L57

Burgasser, A.J., et al. 2002, APJ, 564, 421

Burgasser, A.J., 2006, arXiv:astro-ph/0609556

Butler, R.P., Tinney, C.G., Marcy, G.W., Jones, H.R.A., Penny, A.J. 2001, ApJ, 555, 410

Holman M. J., \& Wiegert, P. A.: 1999, AJ, 117, 621

Knapp, G.R., et al. 2004, AJ, 127, 3553

Lagrange, A.-M., Beust, H., Udry, S., Chauvin, G., \& Mayor, M. 2006, arXiv:astro-ph/0606167 Luhman, K.L., et al. 2006, arXiv:astro-ph/0609464

Mugrauer, M., Neuhäuser, R., Mazeh, T., Guenther, E., \& Fernández, M. 2004a, AN, 325, 718

Mugrauer, M., Neuhäuser, R., Mazeh, T., Alves, J., \& Guenther, E. 2004b, A\&\&A , 425, 249

Mugrauer, M., Neuhäuser, R., Seifahrt, A., Mazeh, T., \& Guenther, E. 2005, A\&\&A , 440, 1051

Mugrauer, M., \& Neuhäuser, R. 2005, MNRAS, 361, L15

Mugrauer, M., Neuhäuser, R., Mazeh, T., \& Guenther, E. 2006a, tafp.conf, 314

Mugrauer, M.,Neuhäuser, R., Mazeh, T., Guenther, E., Fernández, M. 2006b, AN, 327, 321

Mugrauer, M., Seifahrt, A., Neuhaeuser, R., \& Mazeh, T. 2006c, arXiv:astro-ph/0608484

Pichardo, B., Sparke, L.S., \& Aguilar, L.A. 2005, MNRAS, 359, 521

Skrutskie, M.F., et al. 2006, AJ, 131, 1163

Queloz, D., et al. 2000, $A \mathscr{E} A, 354,99$

Weidemann, V. 2000, A\& $A, 363,647$

Worley, C.E., \& Douglass, G.G. 1997, AAPS, 125, 523 